\title{
Slide-Ring Material
}
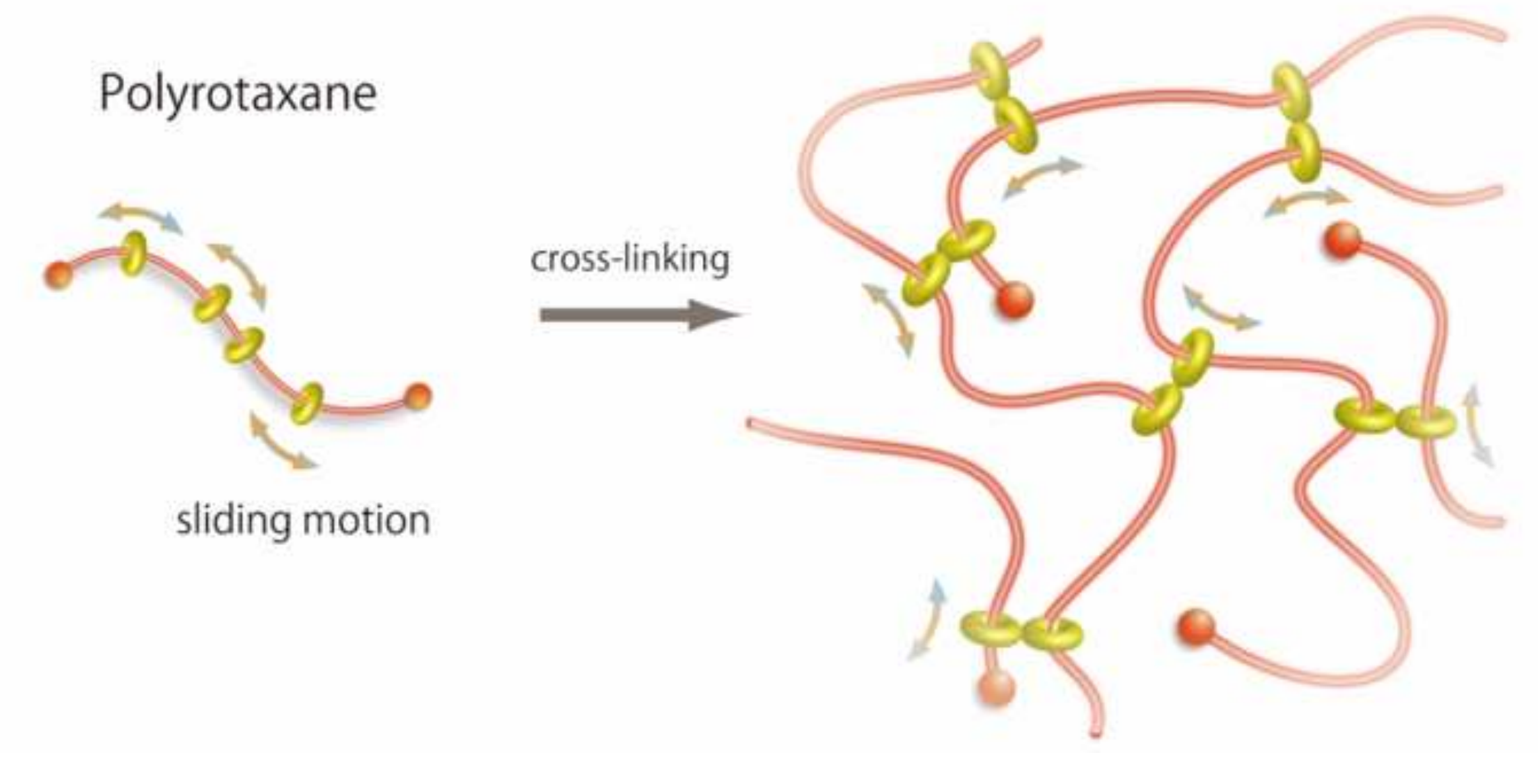

\section{Polyrotaxane}

cross-linking 


\title{
Structure and Dynamics of Polyrotaxane and Slide-Ring Materials
}

\author{
Koichi Mayumi and Kohzo Ito
}

Graduate School of Frontier Sciences, The University of Tokyo, 5-1-5 Kashiwanoha,

Kashiwa, Chiba 277-8561, Japan

Email: kohzo@k.u-tokyo.ac.jp (K. Ito) and kmayumi@molle.k.u-tokyo.ac.jp (K. Mayumi)

Tel.: +81-4-7136-3757

Fax: +81-4-7136-3757

Key Words: Supramolecule, Polyrotaxane, Cyclodextrin, Small-angle scattering, Light scattering 


\begin{abstract}
Polyrotaxane (PR), in which cyclic molecules are threaded into a linear polymer
\end{abstract}

chain, has generated great interest because the sliding and rotation of the cyclic molecules

on the axial polymer chain lead to unique functional nanomaterials with novel dynamical

properties. A typical example of the functional materials is a polyrotaxane network, called

slide-ring (SR) material, prepared by cross-linking the cyclic molecules on different PRs.

The cross-links composed of two cyclic molecules in a shape of figure-of-eight slide along

the polymer chains and the sliding motion gives rise to remarkable physical properties of

the SR materials. In order to understand the unique features of the functional materials

including SR materials and develop novel applications of PR, it is necessary to reveal the

physical properties of PR, especially the sliding motion of the cyclic molecules in PR. In

this article, we review the static structure and molecular dynamics of PR based primarily on

our recent studies. Furthermore, the difference between SR materials and usual chemical

gels in deformation behavior is also described. The findings summarized in this review

indicate the significance of the sliding motion of cyclic molecules characterizing PR and 
SR materials. 


\section{Introduction}

Supramolecular chemistry has developed functional polymers with novel

molecular architectures: supramolecular polymers [1,2]. Supramolecular polymers are

defined as polymeric assemblies of molecular monomers through non-covalent interactions,

such as hydrogen bonding, van der Waals force, topologically interlocking connection, and

so on. In recent years, supramolecular polymers with topologically interlocked structure

have attracted a great attention [3-15], because their components can move with topological

restrictions. A typical example is polyrotaxane (PR) in which a number of cyclic molecules

are threaded onto a linear polymer and are trapped by capping the chain with bulky end

groups (Figure 1) [1]. The cyclic molecules in PR can slide and rotate on the polymer chain,

and this additional kinetic freedom has been utilized to produce functional materials with

novel dynamic properties.

Many attractive functional materials based on PR have been reported. Covering the

axial polymer with the cyclic molecule resulted in unique design of nano-materials, such as

molecular tubes formed by cross-linking adjacent cyclic molecules in a single PR [16] and 
insulated molecular wires incorporating conductive polymers [17]. Sliding and rotational motion of the cyclic molecules on the chain also enables various molecular devices with unique dynamical properties: molecular shuttles [5], drug delivery systems [18], multivalent ligand systems [19,20], energy transfer systems [21], and crystalline polyrotaxane with movable mesogenic side chains [22]. Furthermore, PR has been applied to a novel kind of polymer network known as "slide-ring (SR) material" (Figure 2) [13,23]. SR materials are prepared by cross-linking cyclic molecules on different polyrotaxanes. The cross-links, which comprise two cyclic molecules, can slide along the polymer chains and thereby behave as pulleys to equalize the internal stress in the SR materials. The sliding motion of movable cross-links results in remarkable mechanical properties significantly different from those of conventional polymeric materials with fixed junctions [13-15, 23-26]. The mechanical properties of slide-ring gels are similar to those of biomaterials such as mammalian skin, vessel, and tissues. The slide-ring gels can be used as materials for soft contact lens, artificial skin and vessel And the scratch-resist properties of the slide-ring elastomer without solvent have recently been applied to top coating on a mobile 
phone.

Despite the development of the applications to nanomaterials, basic physical properties of $\mathrm{PR}$, such as conformation and molecular dynamics, have not fully been studied so far. For example, the sliding motion of the cyclic molecules in PR has not been directly observed, and the time scale of the sliding motion is known less. To evaluate the sliding speed in various PRs allow us to control the switching speed from ON state to OFF state in the molecular shuttles, the mechanical properties of the SR materials, and so on. Structure and dynamics of PR, especially the sliding motion, should be revealed to understand potential functions of PR and develop its application to polymeric materials.

In addition, the physical properties of PR are quite interesting topics in polymer physics. The cross-linking in SR materials is regarded as a real example of the slip-link model for the chain-entanglement effect [27], which was previously proposed theoretically. The experimental and theoretical investigations on the dynamics of PR and SR materials may inspire new developments in polymer physics.

In this article, we review recent studies conducted to investigate structure and 
dynamics of PR and SR materials. Section 2 describes the findings about the conformation of PR in dilute and semidilute solutions by sophisticated small-angle neutron scattering (SANS) experiments: (1) the conformation of the axial polymer chain in PR, (2) the distribution of the cyclic molecules on the axial polymer chain, and (3) mechanically interlocked structure between the axial polymer and cyclic molecules. Section 3 introduces the molecular dynamics of PR. Dynamic light scattering (DLS) studies prove that the cyclic molecules in PR can slide on the axial polymer. In addition, neutron spin echo (NSE) technique successfully determines the diffusion constant of PR in $\mathrm{nm}$ and $\mathrm{ns}$ scale. Section 4 summarizes SANS and small-angle X-ray scattering (SAXS) results of SR gels with microscopic homogeneity due to the mobility of cross-links. 


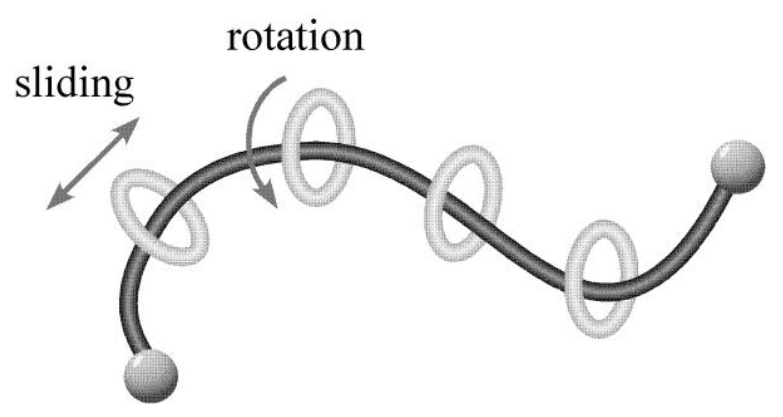

Figure 1. Molecular structure of polyrotaxane (PR), in which cyclic molecules are threaded into a linear polymer chain. The cyclic molecules can slide and rotate on the axial chain. 

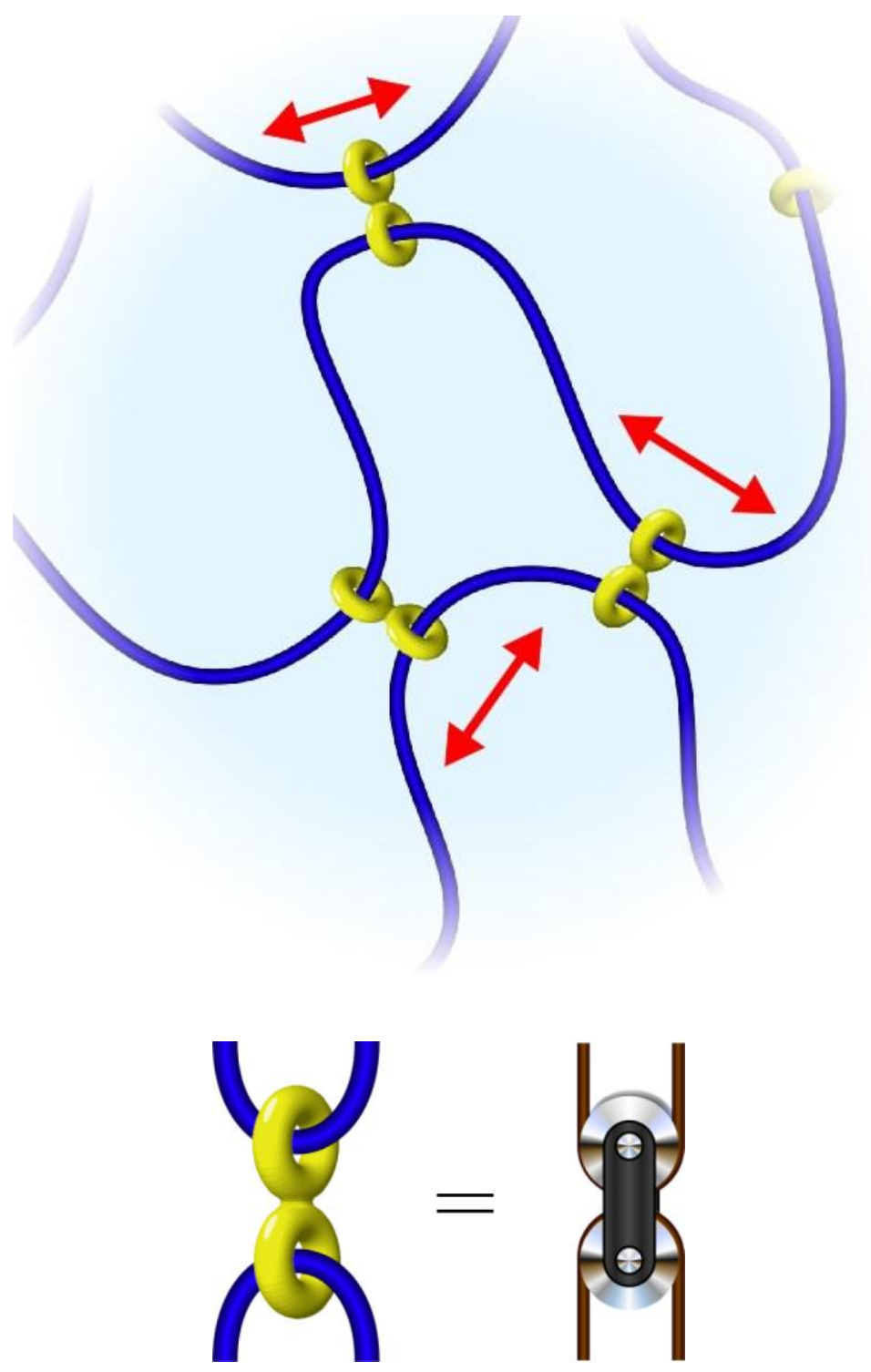

Figure 2. Schematic view of slide ring materials with movable figure-of-eight cross-links.

The cross-links slide along the polymer chains acting like pulleys. 


\section{Static Structure of Polyrotaxane in Solution}

\subsection{Conformation}

The molecular structure of PR, composed of $\alpha$-cyclodextrin (CD) and poly

(ethylene glycol) (PEG) [28], placed on a substrate was directly visualized by scanning tunneling microscopy (STM) [29-30] and atomic force microscopy (AFM) [31]. On the other hand, the conformation of the PR in solution, which is of considerable importance in many application including slide-ring gels, has been investigated by small-angle neutron scattering (SANS) [32-36]. SANS is a very powerful tool to study conformation of polymers: Since SANS experiments first proved the random coil conformation of polymer chains in the melt state [37], static structures of various polymers have been investigated by

SANS [38].

The authors compared hydroxypropylated polyrotaxane (HP-PR) and PEG in dilute solutions by SANS [34]. The hydroxypropylation substantially prevents the hydrogen bonding between adjacent CDs, namely formation of tubular aggregation of CDs in PR $[39,40]$. The coverage ratio of CDs on a PEG chain was approximately $26 \%$. HP-PR and PEG were dissolved into DMSO- $\mathrm{d}_{6}$ at the polymer concentrations of 12 and $6.0 \mathrm{mg} / \mathrm{ml}$, 
respectively. The obtained scattering functions $I(Q)$ for HP-PR and PEG in DMSO- $\mathrm{d}_{6}$ are shown in Figure 3. In high $Q$ regime, $I(Q)$ for PEG is proportional to $Q^{-5 / 3}$, corresponding to the form factor of flexible polymers in good solvent [41]. On the other hand, $I(Q)$ for HP-PR is deviated from $I(\mathrm{Q}) \sim Q^{-5 / 3}$ and the fractal dimension of HP-PR is close to the unity in the high- $Q$ range. This shows that HP-PR is more rigid than PEG. In other words, the inclusion complex formation with CDs stretches the PEG chain, which agrees with the results by other groups $[35,36]$.

In order to estimate the persistence length $l_{\mathrm{p}}$ of HP-PR and PEG, we analyzed the scattering functions by using the generalized Zimm plot [42], given by

$$
I(Q)=\frac{\left(\rho_{\mathrm{P}}-\rho_{\mathrm{S}}\right)^{2}}{N_{\mathrm{A}}} \frac{\Phi(1-\Phi)}{1 /\left(V_{\mathrm{W}} P(Q)\right)+2 A_{2} \Phi}
$$

Here, $N_{A}$ is the Avogadro constant; $\rho_{\mathrm{P} / \mathrm{S}}$, the scattering length density of the polymer/solvent; $\Phi$, the volume fraction of the polymer; $V_{\mathrm{w}}$, the weight average molecular volume; $P(Q)$, the form factor that describes the molecular architecture of the polymer; and $A_{2}$, the second 
virial coefficient. We adopted a function based on the wormlike chain model as the form factor for PR [34]:

$$
P_{\mathrm{PR}}(Q ; L)=\left[(1-\chi(Q ; L)) P_{\text {Debye }}(Q ; L)+\chi(Q ; L) P_{\mathrm{Cyl}}(Q ; L)\right] \Gamma(Q ; L)
$$

where $L$ is the length of the polymer chain, and $\Gamma(Q ; L)$ in eq 2 is the sum of additive factors calculated by Yoshizaki and Yamakawa [43]. $P_{\text {Debye }}(Q ; L)$ is the Debye scattering function for a random coil, and $P_{\mathrm{Cyl}}(Q ; L)$ represents the scattering function for a cylinder with a radius $R$. When $R$ is small enough and negligible, the form factor for $\mathrm{PR}, P_{\mathrm{PR}}(Q)$, reduces to that for the usual wormlike chain model [43]. Since the prefactor $\chi(Q ; L)$ increases with $Q$, $P_{\text {Debye }}(Q ; L)$ dominates in $P_{\mathrm{PR}}(Q ; L)$ in low- $Q$ range and $P_{\mathrm{Cyl}}(Q ; L)$ does in high- $Q$ according to eq. 2 . The cross-over $Q$ from $P_{\text {Debye }}(Q ; L)$ to $P_{\text {Cyl }}(Q ; L)$ gives us the persistence length $l_{\mathrm{p}}$. When $P(Q)$ in eq 1 is given by the wormlike chain model, we have two adjustable parameters, $A_{2}$ and $l_{\mathrm{p}}$, and the other parameters are fixed at the known values in Ref. 34 . The scattering functions for PEG solution is fitted well with eq. 1 and the usual wormlike 
chain model with the radius $R$ equal to zero; however, that for HP-PR deviates from the usual wormlike chain model substantially at high $Q$, as shown by the dashed line in Figure 3. This is because HP-PR has a larger diameter than the PEG chain due to the inclusion of CD molecules. The scattering curve of HP-PR is well described by the modified wormlike chain model with $R$ fixed at the radius of CD, $7 \AA$ [44], as shown by the solid curves in Figure 3.

The fitting parameters obtained are summarized in Table 1. The persistence lengths $l_{\mathrm{p}}$ for PEG and HP-PR are evaluated to be 11 and $44 \AA$, respectively. This means that the inclusion complex formation with CDs increases $l_{\mathrm{p}}$ by four times. The mean square radius of gyration $R_{\mathrm{g}}$ of HP-PR, which is calculated from the obtained $l_{\mathrm{p}}$ according to the wormlike chain model, is $200 \AA$ [34]. This is almost equal to the value measured by static light scattering [45].

The conformation of PR may be affected by the sliding motion of CDs on PEG. If CDs freely slide on PEG in PR in dilute solution, CDs localized within a PEG chain by bulky end-caps tend to diffuse to the outer region away from HP-PR by thermal agitation in 
order to reduce the concentration difference of CDs trapped in PR between the inside and outside of $R_{\mathrm{g}}$ of HP-PR in dilute solution. This pressure from CDs extends $R_{\mathrm{g}}$ of HP-PR with an increase in $l_{\mathrm{p}}$ such as polyelectrolyte solution and gels. The behavior may depend on the PR concentration since such concentration difference disappears in semi-dilute or concentrated solution, where PRs are deeply overlapped with each other. On the other hand, if CDs are fixed at PEG without the sliding motion in PR, $l_{\mathrm{p}}$ ought to be a constant value larger than that of PEG, independently of PR concentration. Such conformational change induced by the sliding motion in PR may be a new aspect in polymer physics. In fact, we found the unique concentration dependence of $l_{\mathrm{p}}$ of PR in solutions due to the change in the sliding motion: the sliding motion of CDs in PR was suppressed as polymer concentration increased [34]. Further studies are underway to confirm the effect of the sliding mode on the PR conformation more directly.

Fleury et al. investigated the conformation of PRs with various coverages from $1 \%$ to $50 \%$ by SANS [35]. They indicated that $R_{\mathrm{g}}$ of PR increased and the conformation changed from a flexible to a rod-like chain with increasing the coverage ratio of CDs on 
PEG. 


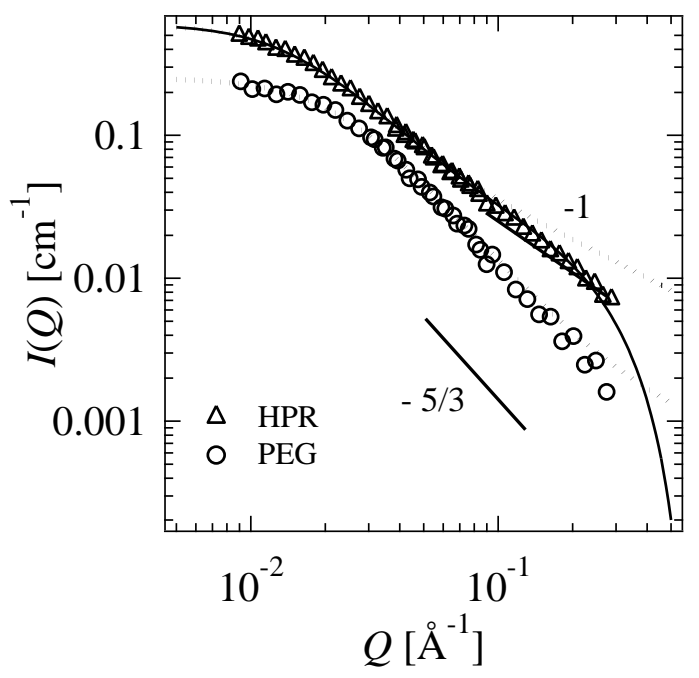

Figure 3. Scattering intensity vs scattering vector $Q$ for HP-PR and PEG in dilute solution.

The dashed and solid curves are derived from eq. 1 with the form factor for the wormlike chain model and for the modified wormlike chain model taking account of the polymer radius, respectively. The data were reproduced from Ref. [34]. 
Table 1. Persistence length, $l_{\mathrm{p}}$, and mean square radius of gyration, $R_{\mathrm{g}}$, for PEG and HP-PR in $\mathrm{DMSO}_{6}$ [34].

\begin{tabular}{|c|c|c|c|}
\hline Sample & $\begin{array}{c}A_{2} \\
\left(10^{-3} \mathrm{~cm}^{3} \mathrm{~mol} \mathrm{~g}^{-2}\right)\end{array}$ & $\begin{array}{c}l_{\mathrm{p}} \\
(\AA)\end{array}$ & $\begin{array}{c}R_{\mathrm{g}} \\
(\AA)\end{array}$ \\
\hline PEG & 2.8 & 11 & 100 \\
\hline HP-PR & 1.9 & 44 & 200 \\
\hline
\end{tabular}




\subsection{Distrubution of Cyclic Molecules on Polymer Chain}

The structural information of PR by SANS experiments reflects a sum of contributions of CDs and PEG composing PR. We investigated the detailed structure of each component in PR solution, especially the spatial distribution of CDs on PEG in PR, by using contrast variation SANS [46]. SANS with contrast variation is a useful method for investigating the structure of multi-component systems such as polymer aggregates formed by diblock copolymers [47], inorganic crystals in the presence of polymer [48], polymer-nanoparticle solutions [49,50], and polymer-clay nanocomposite hydrogels [51-55]. The contrast variation analysis allows us to decompose the observed scattering intensities with different contrasts into the partial scattering functions of each component and to yield detailed information on each structure [48].

The scattering intensity $I(Q)$ of $\mathrm{PR}$ in solution can be expressed using partial scattering functions $S_{\mathrm{ij}}(Q)[48]$ as

$$
I(Q)=\Delta \rho_{\mathrm{C}}^{2} S_{\mathrm{CC}}(Q)+\Delta \rho_{\mathrm{P}}^{2} S_{\mathrm{PP}}(Q)+2 \Delta \rho_{\mathrm{C}} \Delta \rho_{\mathrm{P}} S_{\mathrm{CP}}(Q)
$$

where $\Delta \rho_{\mathrm{i}}$ is the difference between the scattering-length densities $\rho$ of a component $\mathrm{i}$, which is either $\mathrm{C}$ for $\mathrm{CD}$ or $\mathrm{P}$ for $\mathrm{PEG}$, and a solvent. In eq.3, $S_{\mathrm{ij}}$ represents the partial 
scattering function determined by the self-correlation $(\mathrm{i}=\mathrm{j})$ or cross correlation $(\mathrm{i} \neq \mathrm{j})$ of components $\mathrm{i}$ and $\mathrm{j}$. The physical meanings of the partial scattering functions are schematically illustrated in Figure 4. The self-terms $S_{\mathrm{CC}}(Q)$ and $S_{\mathrm{PP}}(Q)$ denote the static structures of $\mathrm{CD}$ and $\mathrm{PEG}$, respectively, and the cross-term $S_{\mathrm{CP}}(Q)$ denotes the pair correlation of $\mathrm{CD}$ and $\mathrm{PEG}$, which reflects the mechanically interlocked connection between the two components. The three unknown partial scattering functions $S_{\mathrm{ij}}$ can be determined by measuring more than three scattering intensities $I(Q)$ 's having different scattering contrasts.

In order to facilitate detailed SANS measurements by contrast variation, we synthesized two types of PRs with different contrasts: h-PR, composed of CD and hydrogenated PEG (h-PEG); and d-PR, composed of CD and deuterated PEG (d-PEG). We prepared solutions of h-PR and d-PR with a volume fraction of $8 \%$ in mixtures of DMSO- $\mathrm{d}_{6}$ and DMSO.

$I(Q)$ 's of h-PR and d-PR obtained by the SANS measurements are shown in Figure 5. The eight $I(Q)$ 's having different contrasts are decomposed into partial scattering 
functions $S_{\mathrm{ij}}(Q)$. The decomposed $S_{\mathrm{CC}}(Q), S_{\mathrm{PP}}(Q)$, and $S_{\mathrm{CP}}(Q)$ are shown in Figure 6. The self-term of $\mathrm{CD}, S_{\mathrm{CC}}(Q)$, is larger than that of PEG, $S_{\mathrm{PP}}(Q)$. This is because the volume fraction of $\mathrm{CD}$ in a $\mathrm{PR}$ is about two point four times as large as that of PEG. Indeed, the ratio of $S_{\mathrm{CC}}(Q)$ to $S_{\mathrm{PP}}(Q)$ is nearly equal to the square of the ratio of the volume fractions. It is important that the cross-term $S_{\mathrm{CP}}(Q)$ for PR is positive and has a magnitude between the magnitudes of the self-terms of $S_{\mathrm{CC}}(Q)$ and $S_{\mathrm{PP}}(Q)$. This indicates an attractive interaction between PEG and CD, which definitely demonstrates a mechanically interlocked connection. This result is consistent with that of 2D-NOESY-NMR studies [56]. Furthermore, the self-term of $\mathrm{CD}, S_{\mathrm{CC}}(Q)$, exhibits almost the same $Q$-dependence as that of PEG, $S_{\mathrm{PP}}(Q)$. This means that $\mathrm{CDs}$ in $\mathrm{PR}$ have the same spatial distribution as $\mathrm{PEG}$ segments in solution. In other words, CDs are not localized but are distributed randomly along the entire PEG chain. These findings obtained by SANS with contrast variation are the first direct evidences that CDs in PR dispersed randomly along the chain in solution.

On the other hand, Travelet et al. suggests that CDs are aggregated and form nano-cylinders in higher concentrated PR solutions [57,58]. The polymer concentration 
may play an important role in the distribution of CDs in PR. The aggregation of CDs in solution also occurs by methylation of PR $[33,59,60]$. Aqueous solutions of methylated PR, consisting of methylated CD and PEG, exhibit sol-gel transition. Methylated CDs are dispersed on PEG chains at low temperature, and they aggregate with increasing temperature due to hydrophobic interaction. The methylated PR can be applied to various smart materials such as thermosensitive slide-ring gels. 


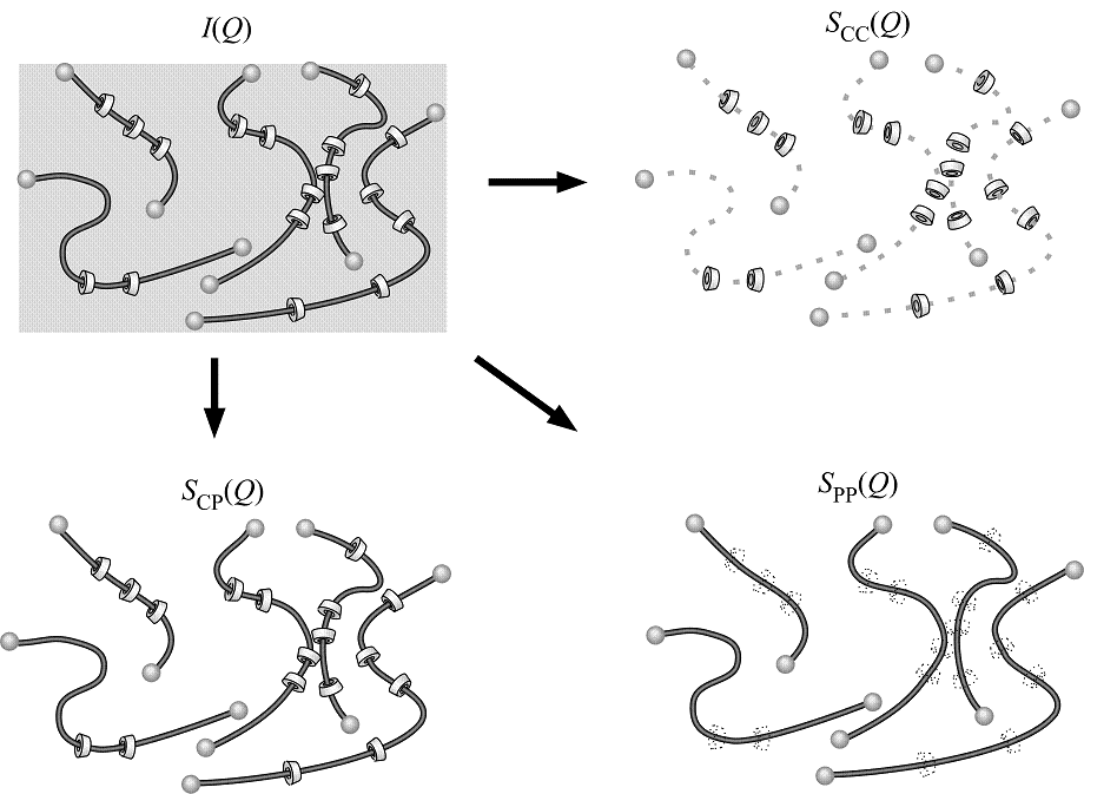

Figure 4. Schematic representations of polyrotaxane in solution showing the decomposition of scattering intensity $I(Q)$ into three partial scattering functions: self-term of PEG, $S_{\mathrm{PP}}(Q)$; self-term of $\mathrm{CD}, S_{\mathrm{CC}}(Q)$; and cross-term of PEG and $\mathrm{CD}, S_{\mathrm{CP}}(Q)$. This figure was reproduced from Ref. [46]. 


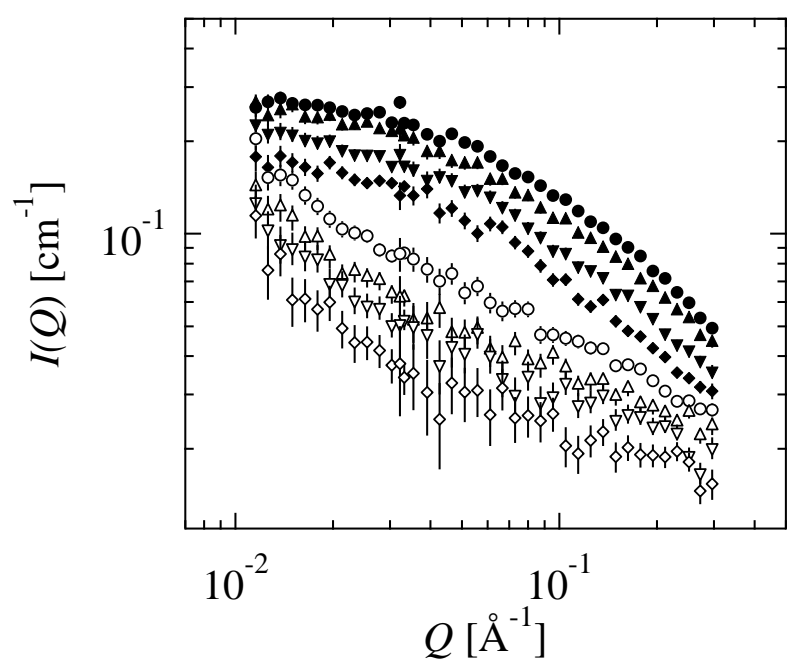

Figure 5. Scattering intensities of h-PR (solid symbols) and d-PR (open symbols) in mixtures of DMSO-d $\mathrm{d}_{6}$ and DMSO. Circles $(\bullet, \circ)$, upward triangles $(\bullet, \Delta)$, downward triangles $(\nabla, \nabla)$, and diamonds $(\bullet, \diamond)$ correspond to $\phi_{\mathrm{DMSO}-\mathrm{d} 6}=1.0,0.95,0.90$, and 0.85 , respectively, where $\phi_{\mathrm{DMSO}-\mathrm{d} 6}$ is the volume fraction of DMSO- $\mathrm{d}_{6}$ in solvent. The errors in $I(Q)$ are \pm one standard deviations of the scattering intensities calculated during the circular averaging. The data were reproduced from Ref. [46]. 


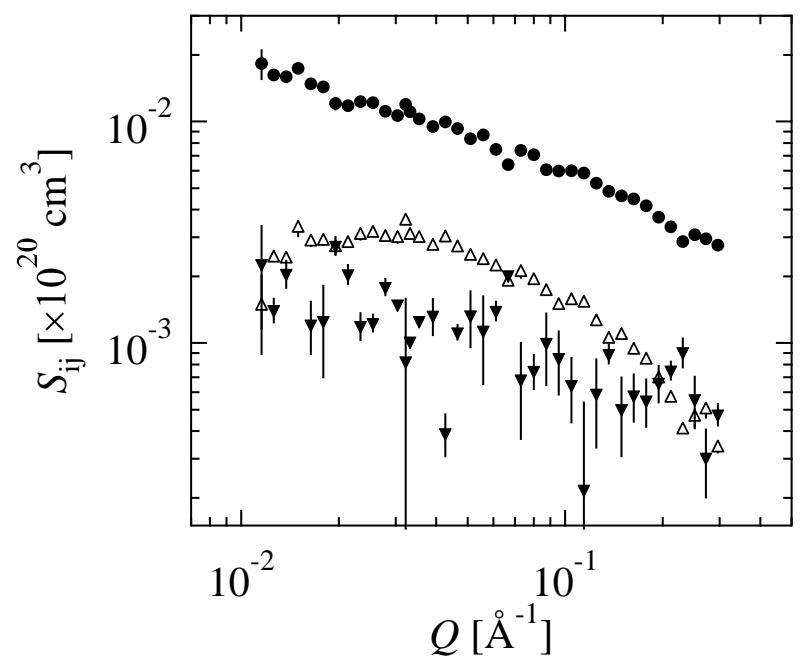

Figure 6. Partial scattering functions of CD-CD, $S_{\mathrm{CC}}(\bullet)$; PEG-PEG, $S_{\mathrm{PP}}(\boldsymbol{v})$; and CD-PEG, $S_{\mathrm{CP}}\left({ }^{\triangle}\right)$. The errors in $S_{\mathrm{ij}}$ are defined by the difference between the observed scattering intensities and the data reconstructed from the estimated scattering contrasts and $S_{\mathrm{ij}}$. The data were reproduced from Ref. [46]. 


\section{Dynamics of Polyrotaxane in Solution}

The most unique feature of PR is the molecular dynamics such as the sliding and rotation of the cyclic molecules on the axial polymer chain, because the dynamics is quite important to understand the functions of polymeric materials using PR. Thus far, some groups have reported nuclear magnetic resonance (NMR) studies on the dynamics of PR. Ceccato et al. performed ${ }^{13} \mathrm{C}-\mathrm{NMR}$ experiments on PR in DMSO- $\mathrm{d}_{6}$ and estimated the rotational correlation time of $\mathrm{CD}$ in PR [61]. From the diffusion-ordered 2D NMR spectroscopy (DOSY) measurements of PR in DMSO- $\mathrm{d}_{6}$, Beckham and co-workers showed that the self-diffusion constants of CD and PEG in PR were equivalent, which verified the

threaded architecture of PR [62]. They also reported the results of solid-state ${ }^{2} \mathrm{H}$ NMR spectroscopy which suggested that PEG segments in PR exhibited faster dynamics than unthreaded PEG [63].

In the following sections, we focus on the translational or sliding motion of the cyclic molecules in PR. The sliding motion is the origin of the excellent physical properties of SR gels described in Section 4. 


\subsection{Dynamic Light Scattering}

We studied the dynamics of PR in solution by dynamic light scattering (DLS)

$[64,65]$. The characteristic decay time distribution functions $G(\Gamma)$ for semidilute polymer

solutions in general show two dynamic modes: self- and cooperative diffusion ones $[27,66]$.

However, PR with the low filling ratio of CDs $(27 \%)$ exhibits three correlation modes

(Figure 7, $0.5 \mathrm{~h}$ ). The third mode with a time constant, $\tau$, of about $0.3 \mathrm{~ms}$ is not detected for

PR with the high filling ratio of $65 \%$, in which CDs are closely packed and the sliding

motion of CDs is suppressed. Therefore the third mode in the sparse PR is assigned to the

sliding motion of CD on PEG. Here, we should note that DLS cannot detect directly the

sliding behavior of a CD molecule since the wavelength of light is much larger than the size

of CD. The third mode observed by DLS would reflect cooperative motion of CDs in PR.

In order to confirm the sliding motion, we investigated the dethreading process of

CDs from PEG in PR by DLS. PR was prepared from $\mathrm{CD}$, polyethylene glycol having

amino groups at both ends (PEG-BA), and 2,4-dinitrofluorobenzene (DNFB) as a capping

molecule according to Harada's method [28,56]: after CDs were threaded into PEG-BA, 
DNFB was reacted with the terminal amino groups of PEG as a bulky end group. The molecular weight of PEG was 35,000 and the filling ratio of CDs on PEG was $27 \%$. When the PR is dissolved into aqueous solutions with high alkali concentration, the end-caps are gradually disconnected from PEG [13].

The diffusion modes of the PR in $1 \mathrm{~N} \mathrm{NaOH}$ aqueous solution change with time as shown in Figure 7. The end groups of the PR are gradually cut off from the PEG chains over several days. After over one day, the dynamic modes observed by DLS reduce to those of the mixture of $\mathrm{CD}$ and PEG in $1 \mathrm{~N} \mathrm{NaOH}$ aqueous solution (Figure 7, $38.8 \mathrm{~h}$ ). It is seen from each DLS spectrum of CD or PEG that the fastest mode reflects the self-diffusion of $\mathrm{CD}$, and the others were the collective and self-diffusion modes of PEG. This indicates that CDs are completely dissociated from the chain after $38.8 \mathrm{~h}$ and namely can slide on PEG in PR. If CDs in PR did not slide but were trapped on the axial chain, the dynamic mode would not change after the removal of the end-caps. As a result, DLS gives us an evidence of the sliding mode of CD on PEG in PR. However, we cannot determine the time scale of the sliding motion because the dissociation process of PR is too fast to detect using DLS. 
Another experimental tool with higher time resolution is needed for it. 


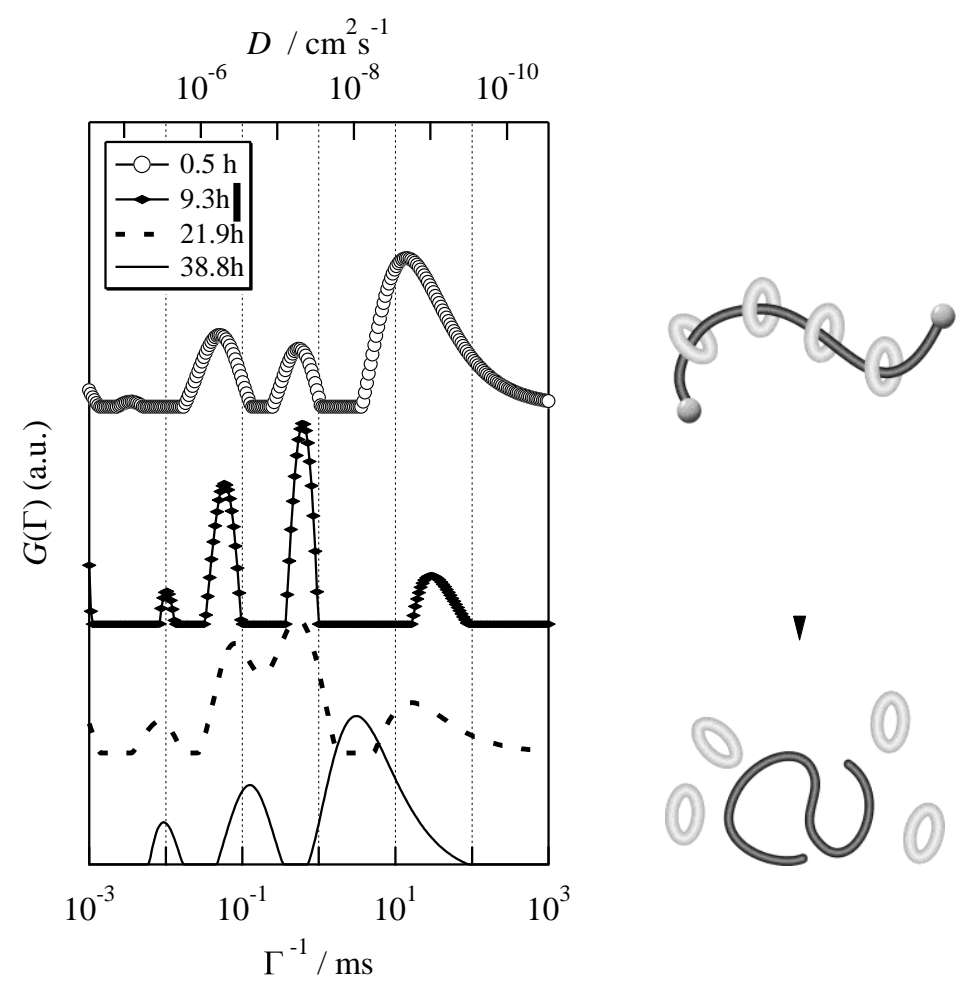

Figure 7. The time evolution of the decay time distribution functions in the dissociation process of $\mathrm{PR}$ in $1 \mathrm{~N} \mathrm{NaOH}$ aqueous solution. 


\subsection{Neutron Spin Echo}

For the direct measurement of the dymaics of CDs in PR, it is necessary to utilize an experimental technique with higher time and spatial resolution than DLS. The authors performed neutron spin echo (NSE) investigations on the molecular dynamics of PR in solution to detect the motion in the spatial scale of a single CD molecule of about $1 \mathrm{~nm}$ in diameter [67]. NSE can measure the dynamics in $\mathrm{nm}$ and $\mathrm{ns}$ scale, and therefore the segmental dynamics of various polymer systems has been studied by NSE [68-70]. It is quite interesting to examine dynamic modes observed for a PR solution in the spatial and time domains of NSE spectroscopy.

In this study, we utilized PEG with a molecular weight of $3.5 \times 10^{4}$ and PR with the filling ratio of $27 \%$. PEG and PR were dissolved in DMSO- $\mathrm{d}_{6}$ with the volume fractions of $10 \%$.

NSE measurements yield the intermediate scattering function $I(Q, t)$. Figure 8 shows the normalized intermediate scattering functions $I(Q, t) / I(Q, 0)$ of $\mathrm{PEG}$ and $\mathrm{PR}$ dissolved in DMSO- $\mathrm{d}_{6}$. The experimental $Q$ range was 0.1 to $0.2 \AA^{-1}$. In order to compare 
the dynamics between PEG and PR quantitatively, we examined the initial decay rate $\Gamma(Q)$ of $I(Q, t)$, calculated from the form $\Gamma(Q)=-(d\{\ln [I(Q, t) / I(Q, 0)]\} / d t)$. The experimental curves were analyzed using a single exponential function $I(Q, t) / I(Q, 0)=\exp (-\Gamma t)$ as the first cumulant, and thus we obtained $\Gamma$. The solid curves in Figure 8 represent the fitting results.

The obtained $\Gamma(Q) / Q^{2}$ for PEG and PR is shown in Figure 9. The results of PEG can be explained by the established theory for linear polymers [27]. $\Gamma(Q) / Q^{2}$ of PEG crosses over from segmental dynamics, i.e., a Zimm mode, to a monomer diffusive mode at approximately $Q=0.15 \AA^{-1}$. However, PR shows a significantly different result from PEG. $\Gamma(Q) / Q^{2}$ of PR is smaller than that of PEG, particularly in the high- $Q$ range. This indicates that the local dynamics of PR is suppressed by threading CDs.

For comparison purposes, the diffusion constant of $\mathrm{CD}$ without an axial polymer in DMSO was estimated by DLS to be $11 \AA^{2} / \mathrm{ns}$, which is almost equal to the monomer diffusion constant of free PEG and one-third as small as that of PR. This suggests that the monomer diffusion constant decreases to one-third due to the mechanically interlocked 
connection between CD and PEG. This may be related to the dimension of diffusion. The motion of CDs in PR is restricted with a one-dimensional chain path of PEG segment, whereas free CDs without an axial polymer can diffuse in three directions.

The contrast variation method described in Section 2.2 can be also applied to NSE and decompose dynamic modes in multi-component systems. In the same manner as the contrast variation SANS, the intermediate scattering function $I(Q, t)$ for $\mathrm{PR}$ in solution is given by the sum of three partial scattering functions corresponding to the motion of the cyclic molecules, dynamics of the axial polymer chain and relative motion between the cyclic molecules and polymer chain, respectively. We will report the results of the CV-NSE experiments on the dynamics of PR in near future [71]. 


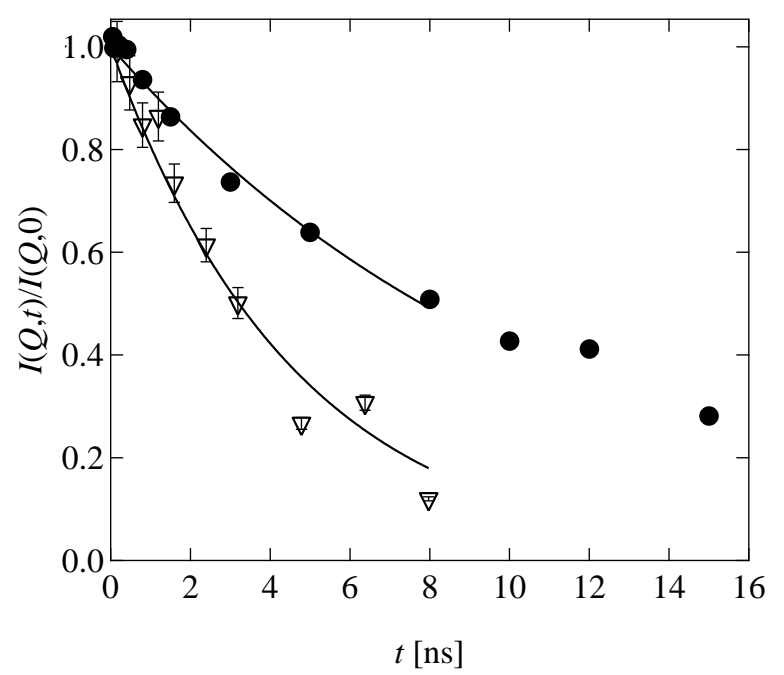

Figure 8. Normalized intermediate scattering functions at $Q=0.16 \AA^{-1}$ for $\operatorname{PR}(\bullet)$ and PEG $(\nabla)$. The curves represent the best-fit results of the first cumulant analysis. The data were reproduced from Ref. [67]. 


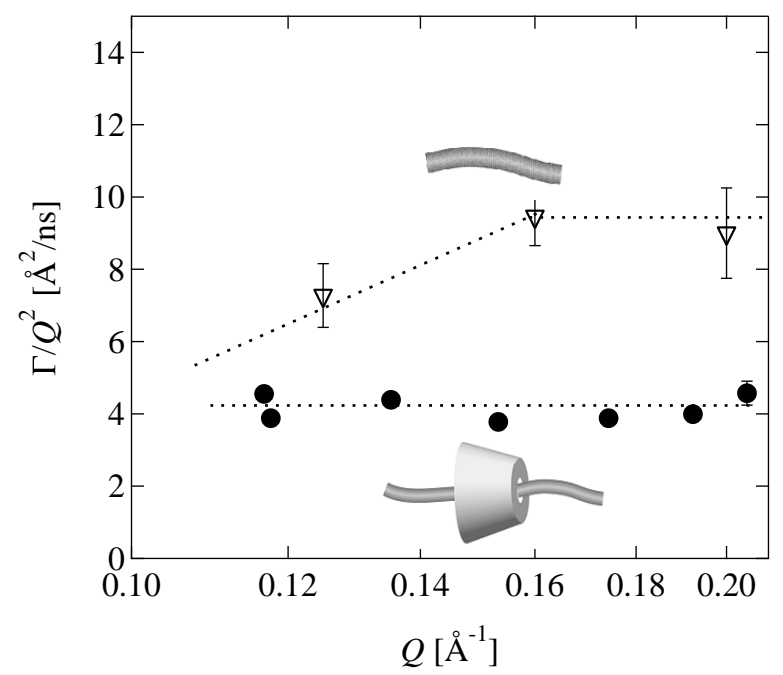

Figure 9. Plot of $\Gamma(Q) / Q^{2}$ as a function of $Q$ for PEG and PR in DMSO- $\mathrm{d}_{6}$. The dashed lines are the guides for the eye. The monomer diffusion constant of PR was approximately one-third as small as that of free CD and PEG. The data were reproduced from Ref. [67]. 


\section{Deformation Mechanism of Slide-Ring Materials}

We have developed a novel type of polymer gel called "slide-ring gel (SR gel)" in

which polymer chains are connected by figure-of-eight cross-links [23], as shown in Figure

2. The double-looped cross-links in SR gels can move along polymer chains to minimize

the local stress in the gels just like pulleys. In order to confirm the pulley effect, we studied

the static structure of the SR gels under uniaxial deformation by means of the small-angle

neutron scattering (SANS) $[65,72,73]$ and X-ray scattering (SAXS) techniques [74].

Shibayama et al. carried out SANS experiments on the deformation mechanism of

the slide-ring gels [72]. In general, it is known that the spatial inhomogeneities increases by

stretching in chemical gels, in which polymer chains are cross-linked by covalent bonds

and the cross-linking junctions are fixed on the polymer chains [75]. As a result, the usual

chemical gels under uniaxial stretching shows the so-called 'abnormal butterfly pattern'

[76-82]. The abnormal butterfly pattern is a two-lobe scattering pattern parallel to the

stretching direction, which is ascribed to the increase of the spatial inhomogeneities in

deformed chemical gels [83-85]. However, as shown in Figure 10, SR gels show a prolate

patterns perpendicular to the scattering direction, called the 'normal butterfly pattern' [72]. 
The normal butterfly pattern is derived from the orientation of polymer chains in homogeneous polymer materials such as polymer solutions in a flow field [86]. Since the polymer chains in SR gels can pass through the cross-links, they would deform like those in polymer solutions in a flow field, and the spatial inhomogeneities of SR gels do not increase in the stretching direction (Figure 11 (b)). This result gives us the strong evidence of the pulley effect in SR gels.

Furthermore, Amemiya et al. performed SAXS investigations on the static structure of deformed SR gels in a good or poor solvent [74]. SR gels in a poor solvent show the abnormal butterfly pattern under uniaxial deformation, while SAXS patterns of SR gels in a good solvent are relatively isotropic at the same deformation ratio, as shown in Figure 12. This is because the movable cross-links aggregate in a poor solvent and the pulley effect does not work. 

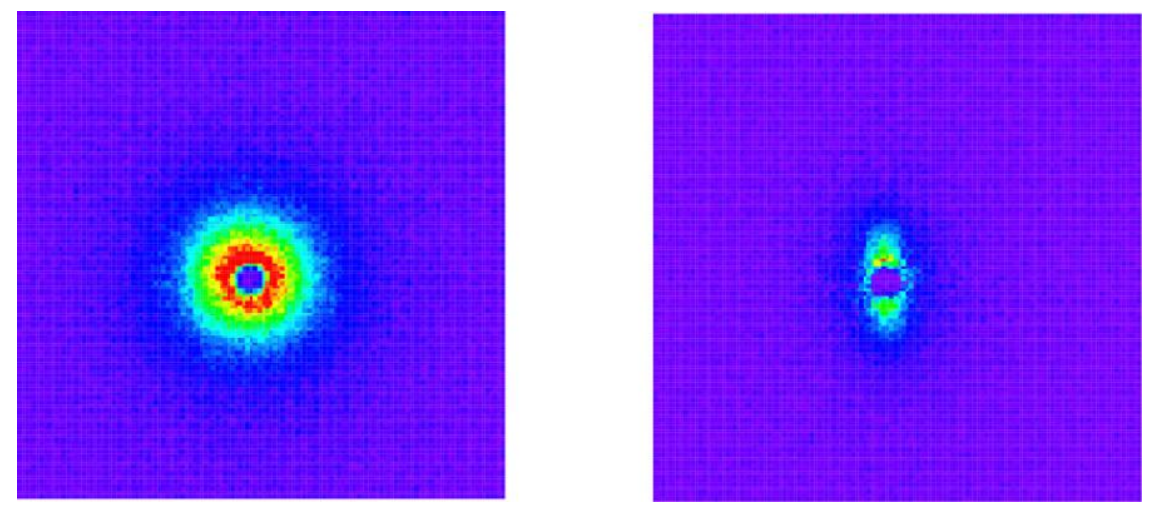

Figure 10. SANS patterns of the SR gel before (left) and after (right) uniaxial deformation 1.8 times in length in the horizontal axis. The normal butterfly pattern perpendicular to the deformed direction is clearly observed. The data were reproduced from Ref. [72]. 
(a) Chemical gel
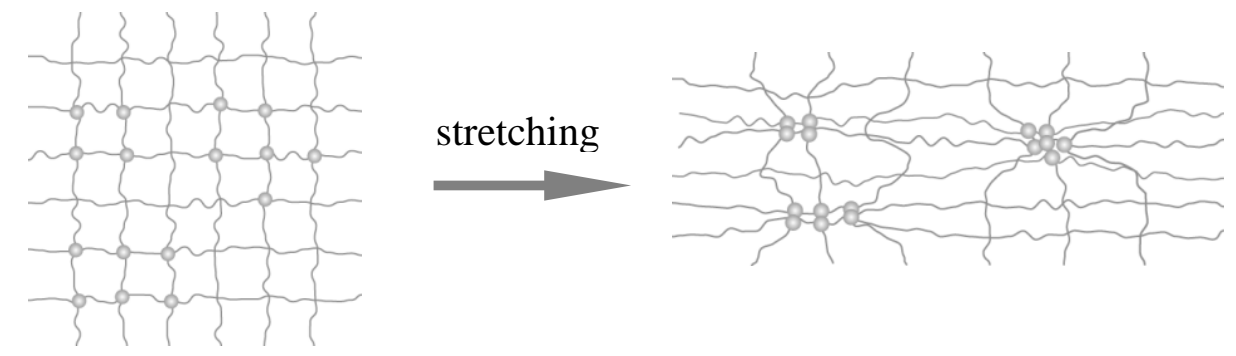

(b) Slide-ring gel
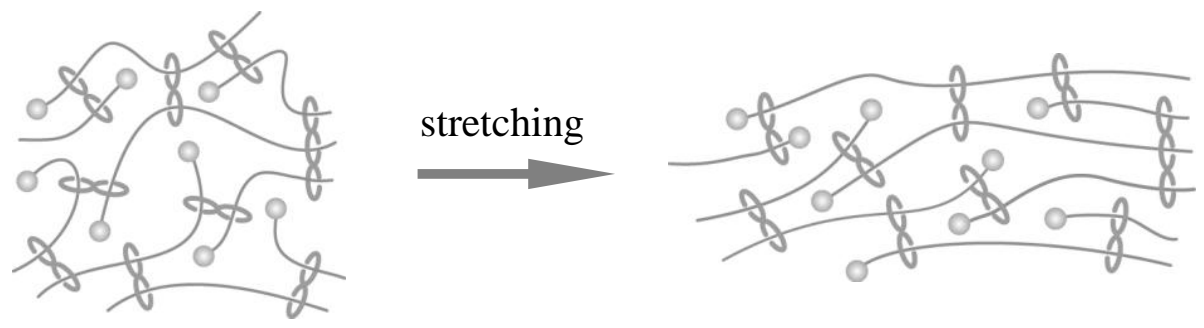

stretching direction

Figure 11. Schematic views showing deformation mechanism for chemical and SR gels.

The figure was reproduced from Ref. [72]. 

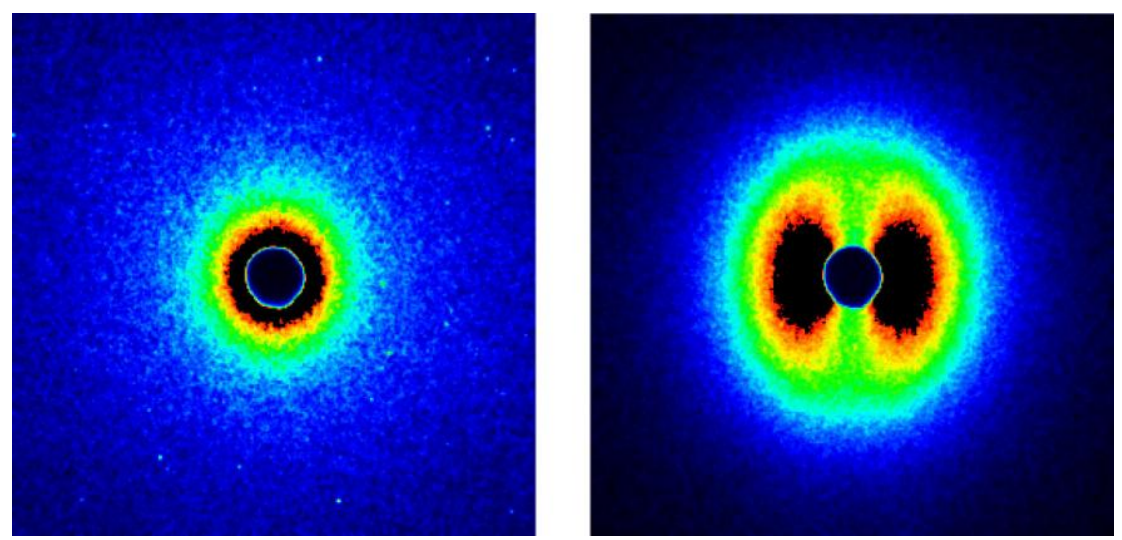

Figure 12. SAXS patterns of the $\mathrm{SR}$ gels with $\mathrm{NaOH}$ (left) and $\mathrm{NaCl}$ (right) aqueous solvents on uniaxial deformation 2 times in length in the horizontal axis. The left figure demonstrates almost the uniform pattern while the right shows the abnormal butterfly one parallel to the deformed direction. The data were reproduced from Ref. [74]. 


\section{Conclusions}

Polyrotaxane (PR), consisting of $\alpha$-cyclodextrins (CDs) and poly(ethylene glycol)

(PEG), is a typical supramolecular polymer with mechanically interlocked structure and has

been applied to various functional nanomaterials including 'slide-ring (SR) material' which

is a cross-linked PR network with movable cross-links. Scattering techniques are very

powerful tools to investigate the physical properties of PR and SR materials. We conducted

SANS studies to determine the static structure of PR in solution. Threading CDs into PEG

stretches the PEG chain and increases the persistence length of PEG. In addition, by using

SANS with the contrast variation method, we obtained the direct evidence of the mechanically interlocked structure of PR and found that the CDs in PR are distributed randomly along the PEG chain.

The most interesting feature of PR is that the CDs in PR can slide along the PEG

chain. The sliding motion of CDs is confirmed by dynamic light scattering (DLS)

experiments on the dissociation process of PR. In order to observe directly the dynamic

behavior of a single CD in PR, we utilized neutron spin echo (NSE) technique, which can 
detect the dynamics in nm and ns scale. NSE determines the diffusion constant of PR to be $4 \AA^{2} /$ ns. This value is about one-third as small as that of free PEG without CDs. This indicates that the topological connection between CD and PEG slows down the local dynamics.

The sliding motion of PR produces the remarkable physical properties of SR materials. SANS and SAXS investigations on the static structure of SR gels under uniaxial deformation revealed that the spatial inhomogeneities of SR gels do not increase in the stretching direction. This result clearly proves that the cross-links in SR gels can slide along the polymer network, and that the internal stress is relaxed via the free movement of the cross-links.

The physical properties of PR and SR materials are quite interesting and significant from the viewpoint of material science as well as polymer physics. It is expected that the findings obtained in this study contribute to novel applications of supramolecular polymers and developments in polymer physics. 


\section{Acknowledgment}

The authors are grateful to Dr. Yasushi Okumura, Mr. Changming Zhao, Dr.

Masatoshi Kidowaki, Dr. Jun Araki, Dr. Yasuhiro Sakai, Dr. Hideaki Yokoyama, Dr.

Naoki Masui, Dr. Toshiyuki Kataoka, Mr. Yusuke Domon, and Ms. Rumiko Kasahara for

their continuous support during the course of this study. The author also gratefully

acknowledges the support of Dr. Hitoshi Endo, Dr. Michihiro Nagao, Dr. Noboru Osaka,

Dr. Takeshi Karino, Dr. Satoshi Okabe, and Dr. Mitsuhiro Shibayama in the SANS and

NSE measurements and Mr. Kentaro Kayashima, Mr. Yuya Shinohara, and Dr. Yoshiyuki

Amemiya in the SAXS measurements. This study is supported by a Grant-in-Aid for

Scientific Research (S) (No. 20221005) and the Core Research for Evolutional Science and

Technology (CREST), Japan Science and Technology Agency. The work at NG5-NSE in

the National Institute of Standards and Technology (NIST), U.S. Department of Commerce,

utilized facilities supported in part by the National Science Foundation under Agreement

No. DMR-0454672. We acknowledge the support of NIST in providing the neutron

research facilities used in this work. The SANS and NSE experiments using SANS-U and 
iNSE were performed with the approval of Institute for Solid State Physics, The University of Tokyo, at the Japan Atomic Energy Agency, Tokai, Japan. 


\section{Reference}

(1) Lehn JM. Supramolecular Chemistry: Concepts and Perspectives. Weinheim: Wiley-VCH; 1995.

(2) Ciferri A. Supramolecular Polymers. New York: Marcel Dekker; 2000.

(3) Sauvage JP, Dietrich-Buchecker C. Molecular Catenanes, Rotaxanes, and Knots. Weinheim: Wiley-VCH; 1999.

(4) Huang F, Gibson HW. Prog Polym Sci 2005; 30: 982-1018.

(5) Nepogodiev SA, Stoddart JF. Chem Rev 1998; 98: 1959-76.

(6) Raymo FM, Stoddart JF. Chem Rev 1999; 99: 1643-63.

(7) Wenz G, Han BH, Muller A. Chem Rev 2006; 106: 782-817.

(8) Harada A, Hashidzume A, Takashima Y. Adv Polym Sci 2006; 201: 1-43.

(9) Takata T, Kihara N, Furusho Y. Adv Polym Sci 2004; 171: 1-76.

(10) Choi HS, Yui N. Prog Polym Sci 2006; 31: 121-44.

(11) Loethen S, Kim JM, Thompson DH. Polymer Reviews 2007; 47: 383-418.

(12) Tonelli AE. Polymer 2008; 49: 1725-36. 
(13) Araki J, Ito K. Soft matter 2007; 3: 1456-73.

(14) Ito K. Polym J 2007; 39: 489-99.

(15) Ito K. Curr Opin Solid State Mater Sci: in press.

(16) Harada A, Li J, Kamachi M. Nature 1993; 364: 516-8.

(17) Frampton MJ, Anderson HL. Angew Chem Int Ed 2007; 46: 1028-64.

(18) Ooya T, Yui N. J Controlled Release 1999; 58: 251-69.

(19) Yui N, Ooya T. Chem Eur J 2006; 12: 6730-7.

(20) Ooya T, Eguchi N, Yui N. J Am Chem Soc 2003; 125: 13016-7.

(21) Tamura M, Ueno A. Bull Chem Soc Jpn 2000; 73: 147-54.

(22) Kidowaki M, Nakajima T, Araki J, Inomata A, Ishibashi H, Ito K. Macromolecules 2007; 40: 6859-62.

(23) Okumura Y, Ito K. Adv Mater 2001; 13: 485-7.

(24) Fleury G, Schlatter G, Brochon C, Hadziioannou G. Polymer 2005; 46: 8494-501.

(25) Fleury G, Schlatter G, Brochon C, Hadziioannou G. Adv Mater 2006; 18: 2847-51.

(26) Fleury G, Schlatter G, Brochon C, Travelet C, Lapp A, Lindner P, Hadziioannou G. 
Macromolecules 2007; 40: 535-43.

(27) Doi M, Edwards SF. The Theory of Polymer Dynamics. Oxford: Oxford University Press; 1986.

(28) Harada A, Li J, Kamachi M. Nature 1992; 356: 325-7.

(29) Yoshida K, Shimomura T, Ito K, Hayakawa R. Langmuir 1999; 15: 910-3.

(30) Shigekawa H, Miyake K, Sumaoka J, Harada A, Komiyama M. J Am Chem Soc 2000;

122: 5411-2.

(31) van den Boogaard M, Bonnet G, Van't Hof P, Wang Y, Brochon C, van Hutten P, Lapp A, Hadziioannou G. Chem Mater 2004; 16: 4383-5.

(32) Karino T, Okumura Y, Ito K, Shibayama M. Macromolecules 2004; 37: 6177-82.

(33) Karino T, Okumura Y, Zhao C, Kidowaki M, Kataoka T, Ito K, Shibayama M.

Macromolecules 2006; 39: 9435-40.

(34) Mayumi K, Osaka N, Endo H, Yokoyama H, Sakai Y, Shibayama M, Ito K. Macromolecules 2008; 41: 6580-5.

(35) Fleury G, Brochon C, Schlatter G, Bonnet G, Lapp A, Hadziioannou G. Soft Matter 
$2005 ; 1: 378-85$.

(36) Jarroux N, Guegan P, Cheradame H, Auvray L. J Phys Chem B 2005; 109: 23816-22.

(37) Ballard DGH, Wignall GD, Schelten J. Eur Polym J 1973; 9: 965-9.

(38) Higgins JS, Benoit HC. Polymers and Neutron Scattering. Oxford: Clarendon Press;

1994.

(39) Araki J, Ito K. J Polym Sci Part A: Polym Chem 2006; 44: 6312-23.

(40) Ooya T, Yui N. Macromol Chem Phys 1998; 199: 2311-20.

(41) Rubinstein M, Colby RH. Polymer Physics. Oxford: Oxford University Press; 2003.

(42) Stellbrink J, Willner L, Jucknischke O, Richter D, Lindner P, Fetters LJ, Huang S.

Macromolecules 1998; 31: 4189-97.

(43) Yoshizaki T, Yamakawa H. Macromolecules 1980; 13: 1518-25.

(44) Lichtenthaler FW, Immel S. Tetrahedron: Asymmetry 1994; 5: 2045-60.

(45) Kume T, Araki J, Sakai Y, Mayumi K, Kidowaki M, Yokoyama H, Ito K. J Phys Conf

Ser: in press. 
(46) Mayumi K, Endo H, Osaka N, Yokoyama H, Nagao M, Shibayama M, Ito K. Macromolecules 2009; 42: 6327-9.

(47) Richter D, Schneiders D, Monkenbusch M, Willner L, Fetters LJ, Huang JS, Lin M, Mortensen K, Farago B. Macromolecules 1997; 30: 1053-68.

(48) Endo H, Schwahn D, Cölfen H. J Chem Phys 2004; 120: 9410-23.

(49) Shcmidt G., Nakatani AI, Butler PD, Han CC. Macromolecules 2002; 35: 4725-32.

(50) Hecht AM, Horkay F, Geissler E. Phys Rev E 2001; 64: 041402.

(51) Shibayam M, Karino T, Miyazaki S, Okabe S, Takehisa T, Haraguchi K. Macromolecules 2005; 38: 10772-81.

(52) Miyazaki S, Karino T, Endo H, Haraguchi K, Shibayama M. Macromolecules 2006; 39: 8112-20.

(53) Endo H, Miyazaki S, Haraguchi K, Shibayama M. Macromolecules 2008; 41: 5406-11. 
(54) Osaka N, Endo H, Nishida T, Suzuki T, Li HJ, Haraguchi K, Shibayama M. Phys Rev E 2009; 79: 060801.

(55) Nishida T, Endo H, Osaka N, Li HJ, Haraguchi K, Shibayama M. Phys Rev E 2009; 80: 030801.

(56) Harada A, Li J, Nakamitsu T, Kamachi M. J Org Chem 1993; 58: 7524-8.

(57) Travelet C, Schlatter G, Hebraud P, Brochon C, Lapp A, Anokhin DV, Ivanov DA, Gaillard C, Hadziioannou G. Soft Matter 2008; 4: 1855-60.

(58) Travelet C, Schlatter G, Hebraud P, Brochon C, Lapp A, Hadziioannou G. Langmuir 2009; 25: 8723-34.

(59) Kidowaki M, Zhao C, Kataoka T, Ito K. Chem Commun 2006; 39: 4102-3.

(60) Kataoka T, Kidowaki M, Zhao C, Minamikawa H, Shimizu T, Ito K. J Phys Chem B 2006; 110: 24377-83.

(61) Ceccato M, LoNostro P, Rossi C, Bonechi C, Donati A, Baglioni P. J Phys Chem B 1997; 101: 5094-9. 
(62) Zhao T, Beckham HW. Macromolecules 2003; 36: 9859-65.

(63) Girardeau TE, Leisen J, Beckham HW. Macromol Chem Phys 2005; 206: 998-1005.

(64) Zhao C, Domon Y, Okumura Y, Okabe S, Shibayama M, Ito K. J Phys: Condens

Matter 2005; 17: S2841-6.

(65) Shibayama M, Karino T, Domon Y, Ito K. J Appl Cryst 2007; 40: s43-7.

(66) de Gennes PG. Scaling Concepts in Polymer Physics. New York: Cornell University Press; 1979.

(67) Mayumi K, Nagao M, Endo H, Osaka N, Shibayama M, Ito K. Physica B 2009; 404: $2600-2$.

(68) Mezei F. Neutron spin echo spectroscopy; Lecture Notes in Physics 128. Heidelberg Germany New York: Springer; 1980.

(69) Ewen B, Richter D. Adv Polym Sci 1997; 134: 1-128.

(70) Richter D, Monkenbusch M, Arbe A, Colmenero J. Adv Polym Sci 2005; 174: 1-221.

(71) Mayumi K. et al. in preparation.

(72) Karino T, Okumura Y, Zhao C, Kataoka T, Ito K, Shibayama M. Macromolecules 
2005; 38: 6161-7.

(73) Karino T, Shibayama M, Ito K. Physica B 2006; 385-386: 692-6.

(74) Shinohara Y, Kayashima K, Okumura Y, Zhao C, Ito K, Amemiya Y. Macromolecules 2006; 39: 7386-91.

(75) Shibayama M. Macromol Chem Phys 1998; 199: 1-30.

(76) Bastide J, Leibler L. Macromolecules 1988; 21: 2647-9.

(77) Bastide J, Leibler L, Prost J. Macromolecules 1990; 23: 1821-5.

(78) Mendes E, Lindner P, Buzier M, Boue F, Bastide J. Phys Rev Lett 1991; 66: 1595-8.

(79) Zielinski F, Buzier M, Lartigue C, Bastide J. Prog Colloid Polym Sci 1992; 90: $115-30$.

(80) Rouf C, Bastide J, Pujol JM, Schosseler F, Munch JP. Phys Rev Lett 1994; 73: 830-3.

(81) Ramzi A, Zielinski F, Bastide J, Boue F. Macromolecules 1995; 28: 3570-87.

(82) Shibayama M, Kawakubo K, Ikkai F, Imai M. Macromolecules 1998; 31: 2586-92. 
(83) Onuki A. J Phys II 1992; 2: 45-61.

(84) Panyukov S, Rabin Y. Macromolecules 1996; 29: 7960-75.

(85) Rabin Y, Panyukov S. Macromolecules 1997; 30: 301-12.

(86) Moses E, Kume T, Hashimoto T. Phys Rev Lett 1994; 72: 2037-40. 


\section{For Table of Contents Use Only}

Structure and Dynamics of Polyrotaxane and Slide-Ring Materials

Koichi Mayumi, and Kohzo Ito

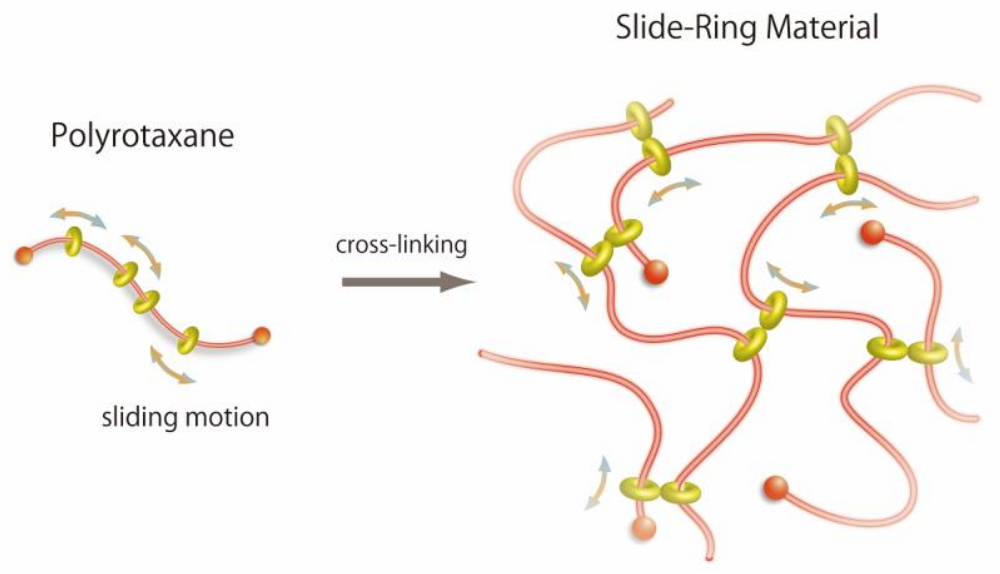

